OPEN ACCESS

Edited by:

David J. Seiffge,

University Hospital Bern, Switzerland

Reviewed by:

Jukka Putaala,

Helsinki University Central

Hospital, Finland

Alexandros A. Polymeris,

University Hospital of

Basel, Switzerland

${ }^{*}$ Correspondence:

Yang-Ha Hwang

yangha.hwang@gmail.com

Specialty section:

This article was submitted to

Stroke,

a section of the journal

Frontiers in Neurology

Received: 11 March 2021

Accepted: 03 May 2021

Published: 22 June 2021

Citation:

Gwak D-S, Choi WC, Kim Y-W

Kim Y-S and Hwang Y-H (2021)

Impact of Left Atrial Appendage Morphology on Recurrence in Embolic

Stroke of Undetermined Source and Atrial Cardiopathy.

Front. Neurol. 12:679320.

doi: 10.3389/fneur.2021.679320

\section{Impact of Left Atrial Appendage Morphology on Recurrence in Embolic Stroke of Undetermined Source and Atrial Cardiopathy}

\author{
Dong-Seok Gwak ${ }^{1}$, WooChan Choi ${ }^{1}$, Yong-Won Kim ${ }^{1,2}$, Yong-Sun Kim ${ }^{3,4}$ and \\ Yang-Ha Hwang ${ }^{1,2 *}$
}

${ }^{1}$ Department of Neurology, Kyungpook National University Hospital, Daegu, South Korea, ${ }^{2}$ Department of Neurology, School of Medicine, Kyungpook National University, Daegu, South Korea, ${ }^{3}$ Department of Radiology, Kyungpook National University Hospital, Daegu, South Korea, ${ }^{4}$ Department of Radiology, School of Medicine, Kyungpook National University, Daegu, South Korea

Background: The left atrial appendage ( $\angle A A)$ is a major source of thrombus and non-chicken wing (CW). LAA morphology is a risk factor for embolic events in atrial fibrillation. However, the association of non-CW morphology with embolic stroke recurrence is unknown in patients with embolic stroke of undetermined source (ESUS) and atrial cardiopathy.

Methods: We conducted retrospective analyses using a prospective institutional stroke registry (2013-2017). Patients with ESUS and atrial cardiopathy were enrolled. Atrial cardiopathy was diagnosed if an increased left atrial diameter (>40 mm, men; $>38 \mathrm{~mm}$, women), supraventricular tachycardia, or LAA filling defect on computed tomography (CT) were present. Patients admitted $>24 \mathrm{~h}$ after onset were excluded. LAA morphology was evaluated using CT and categorized into CW vs. non-CW types. The primary outcome was embolic stroke recurrence. Multivariable Cox proportional hazards models were used to examine the independent association between LAA morphology and outcome.

Results: Of 157 patients, 81 (51.6\%) had CW LAA morphology. The median followup was 41.5 (interquartile range 12.3-58.5) months corresponding to 509.8 patient years. In total, 18 participants experienced embolic stroke recurrences (3.80 per 100 patient-years). Non-CW morphology was more associated with embolic stroke recurrence than $\mathrm{CW}$ morphology (hazard ratio $(\mathrm{HR}), 3.17$; 95\% confidence interval (Cl), 1.13-8.91; $p=0.029$ ). After adjusting for $\mathrm{CHA}_{2} \mathrm{DS}_{2}$-VASc score and number of potential embolic sources, non-CW morphology showed an independent association with outcome (adjusted HR, 2.90; 95\% Cl, 1.02-8.23; $p=0.045$ ).

Conclusions: The LAA morphology types may help identify high risk of embolic stroke recurrence in ESUS with atrial cardiopathy. LAA morphology in atrial cardiopathy may provide clues for developing therapies tailored to specific mechanisms.

Keywords: recurrence, embolic strokes, atrial appendage, cerebral infarction, CT scan 


\section{INTRODUCTION}

The concept of embolic stroke of undetermined source (ESUS) assumed that most cryptogenic strokes are thromboembolic and could benefit from anticoagulation (1). However, the failure of the two recent clinical trials to demonstrate a reduction in stroke recurrence with anticoagulants for patients with ESUS facilitates the conceptual change that ESUS comprises heterogeneous subgroups $(2,3)$. One of the subgroups of ESUS is atrial cardiopathy, which leaves atrial substrates with structural, functional, or electrical remodeling preceding clinical atrial fibrillation (AF) (4). Abnormal atrial substrates per se could be thrombogenic even in the absence of AF (5-9). Several markers of atrial cardiopathy are associated with subsequent stroke events $(10,11)$. Moreover, anticoagulant therapy may be beneficial for a subset of these patients with high-risk characteristics for thromboembolism including an enlarged left atrium (12) or elevated N-terminal pro-B-type natriuretic peptides (13).

Another potential biomarker of embolic risk in atrial cardiopathy is the left atrial appendage (LAA) morphology. LAA varies significantly in shape and has usually been categorized into four distinct morphologies: chicken wing $(\mathrm{CW})$, windsock, cactus, and cauliflower (14-16). LAA is a major source of thrombus in patients with AF (17), and, compared with CW morphology, non-CW LAA morphology is related to embolic events $(14,18)$. However, the association of non-CW morphology with recurrent embolic stroke is unknown in patients with ESUS and atrial cardiopathy. Thus, this study aimed to determine whether non-CW morphology increases the recurrence risk of embolic stroke in this patient population.

\section{MATERIALS AND METHODS}

\section{Study Population}

Consecutive stroke patients were retrospectively reviewed from the prospective institutional stroke registry database. The inclusion criteria for this study are as follows: (1) admission to Kyungpook National University Hospital from April 2013 to December 2017, (2) ESUS defined by the Cryptogenic Stroke/ESUS International Working Group (1), and (3) atrial cardiopathy. This is defined as the increased left atrial diameter ( $>40 \mathrm{~mm}$ for men and $>38 \mathrm{~mm}$ for women), supraventricular tachycardia/subclinical AF, or an LAA filling defect on cerebral computed tomography angiography (CCTA) (19-23). However, patients who were admitted $>24 \mathrm{~h}$ of symptom onset and who did not have a CCTA extending to the lower limit of the scan range to the diaphragm were excluded. Finally, 157 patients were eligible for analysis (Figure 1). The study protocol was approved by the local institutional review board (IRB approval number: KNUH 2020-10-018). Written informed consent was waived because of the retrospective nature of the study and the anonymity of study subjects.

\section{Clinical Data Collection}

Baseline demographics for study participants were extracted from the institutional stroke registry database or electronic medical records. Potential embolic sources, including arterial atherosclerotic disease (presence of any ipsilesional carotid stenosis of $<50 \%$ or aortic arch atherosclerosis), left ventricular dysfunction (presence of low left ventricular ejection fraction of $30-35 \%$ or regional wall motion abnormality reported in the echocardiogram or presence of heart failure history), cardiac valve disease (presence of moderate to severe stenosis or regurgitation of aortic or mitral valve identified in the echocardiogram), patent foramen ovale (any grade; detected on the echocardiogram), and cancer (history of cancer, except skin cancer, at baseline), were collected $(20,21)$. The $\mathrm{CHA}_{2} \mathrm{DS}_{2}$-VASc score, which is a well-validated tool for stratifying the risk of stroke in patients with AF or ESUS, was calculated (sum of the point values of variables of congestive heart failure, hypertension, age ( 1 point, $65-74 ; 2$ points, $\geq 75$ ), diabetes mellitus, prior stroke or transient ischemic attack (2 points), vascular disease, and female sex $(24,25)$. Moreover, echocardiographic findings, LAA filling defect on CCTA, supraventricular tachycardia (paroxysmal tachyarrhythmia with narrow QRS complex and regular ventricular response which is initiated by atrial or atrioventricular node) (26) or subclinical $\mathrm{AF}$ ( $\mathrm{AF}$ detected during cardiac monitoring lasting $<6 \mathrm{~min}$ ) (20), and AF detection during the follow-up period were collected. Transesophageal echocardiography (TEE) parameters, including LAA emptying velocity (the peak velocity of the flow out of the LAA) (18) and spontaneous echogenic contrast (SEC; visually diagnosed by the presence of characteristic swirling smoke-like echoes distinguished from background white noise) (27) on LAA, were also collected. The primary outcome was embolic stroke recurrence defined as sudden onset focal neurological deficits lasting for $>24 \mathrm{~h}$ with evidence of acute non-lacunar brain infarct and no relevant arterial stenosis on brain imaging. The secondary outcomes were ischemic stroke recurrence, any stroke (ischemic or hemorrhagic) recurrence, and all-cause mortality.

\section{Assessment of LAA Morphology}

According to the institutional protocol, CCTA was obtained on admission to the emergency department in patients with suspected acute ischemic stroke within $24 \mathrm{~h}$ of symptom onset (The detailed imaging protocol is described in Supplementary Figure 1). The CCTA source images were then post-processed to create three-dimensional volumetric reconstructions of LAA using an open-source program for biomedical research, 3D Slicer (version 4.10.2., http://www.slicer. org). Two vascular neurologists blinded to outcomes (Gwak D-S and Choi WC) visually analyzed LAA morphology according to the previously reported classifications (14-16) and assigned it to two classes (CW vs. non-CW type-windsock, cactus, and cauliflower type; Figure 2). The CW type is characterized by an obvious bend $<100^{\circ}$ in the proximal or middle part of the dominant lobe; windsock morphology presents one dominant lobe of sufficient length with or without secondary lobes with bending $>100^{\circ}$; cactus presents a dominant central lobe with one or more secondary lobes extending from the central lobe; and cauliflower is characterized by the limited overall length with complex internal structures. The discrepancies in LAA morphology were resolved by consensus (Kappa index, 0.770 ). 


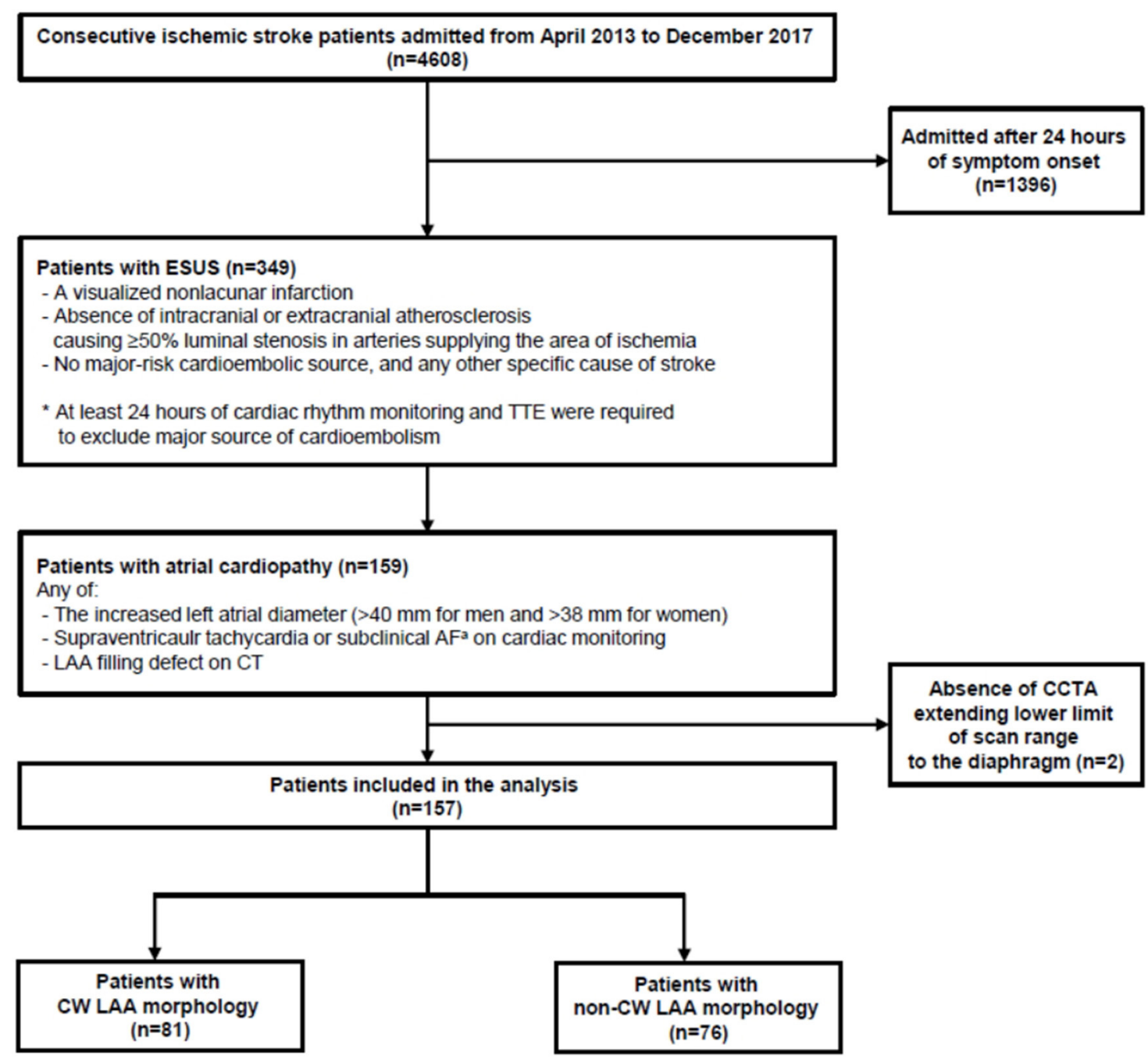

FIGURE 1 | Flowchart of patient screening and enrollment. ESUS, embolic stroke of undetermined source; TTE, transthoracic echocardiography; AF, atrial fibrillation; LAA, left atrial appendage; CT, computed tomography. ${ }^{a}$ Defined as atrial fibrillation for $<6 \mathrm{~min}$.

\section{Statistical Analysis Plan}

Time-to-event data were summarized by the number of events per patient-years of exposure and examined using the KaplanMeier curves, with comparisons between the groups with CW and non-CW morphologies made with the log-rank test. Univariate and multivariable Cox proportional hazards regression analyses were performed to examine the independent association of non-CW LAA morphology with outcomes. Several multivariable models were constructed with prespecified covariates or variables imbalanced between the groups with $\mathrm{CW}$ and non-CW morphologies due to the small sample size. Model 1 was adjusted for age, sex, and baseline National Institute of Health Stroke Scale score. Model 2 was adjusted for variables of potential risks for outcomes $\left(\mathrm{CHA}_{2} \mathrm{DS}_{2}\right.$-VASc score and number of potential embolic sources). Model 3 included the imbalanced baseline characteristics and cardiac biomarkers between the two groups with $p<0.10$. Associations were reported as hazard ratios (HRs) and 95\% confidence intervals (CIs). Those who were available for TEE parameters including LAA emptying velocity and SEC on LAA were subsequently analyzed between the two groups. A two-sided $p<0.05$ was considered statistically significant. All statistical analyses were performed using SPSS software (version 25.0, IBM SPSS, Armonk, NY, USA). The Kaplan-Meier curves were generated by using GraphPad Prism (version 9.0.0, GraphPad Software, Inc., La Jolla, CA, USA).

\section{RESULTS}

The cohort dataset comprised 349 patients with ESUS. Among them, 157 patients with atrial cardiopathy were included. Of the patients with different LAA morphologies, 81 (51.6\%), 33 (21.0\%), 25 (15.9\%), and 18 (11.5\%) were categorized as CW, windsock, cactus, and cauliflower, respectively. The baseline demographics and clinical characteristics were compared 


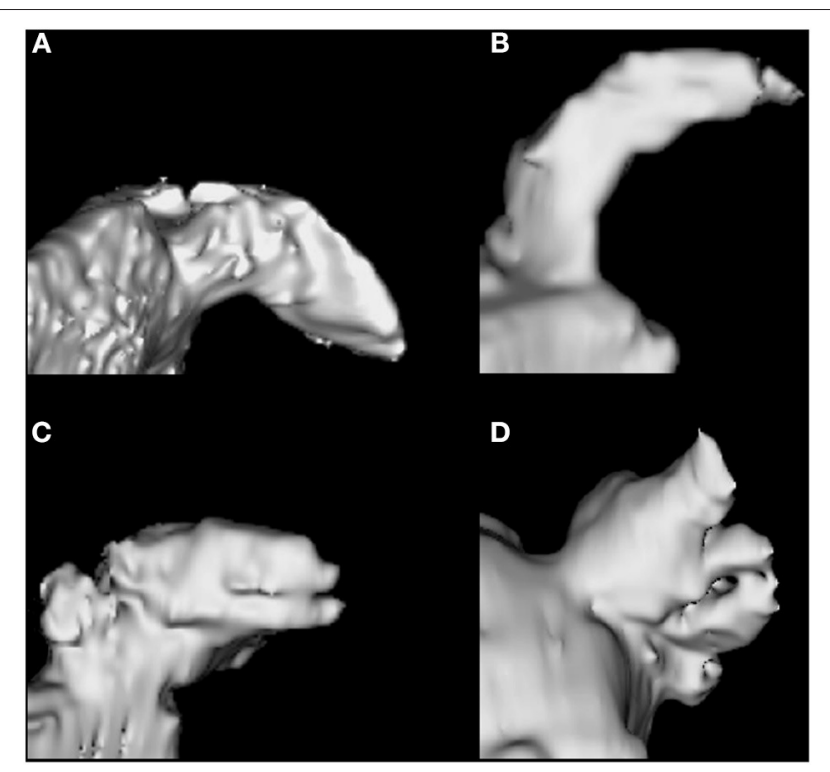

FIGURE 2 | Left atrial appendage morphology types. Chicken wing (A), Windsock (B), Cactus (C), Cauliflower (D).

between patients with CW and non-CW LAA morphologies (Table 1). The mean age was $69.5 \pm 11.6$ years with $58 \%$ were men. Other variables were not statistically different between the two groups except for hyperlipidemia (43.4 vs. $27.2 \%, p=0.033$ ). Factors associated with atrial cardiopathy are shown in Table 2. No significant differences in cardiac markers exist between the two groups.

The median follow-up was 41.5 (interquartile range, 12.358.5) months corresponding to 509.8 patient years. In total, $18(11.5 \%)$ embolic stroke recurrences, 21 (13.4\%) ischemic stroke recurrences, $23(14.6 \%)$ any stroke recurrences, and 13 (8.3\%) deaths corresponded to 3.80, 4.47, 4.90, and 2.55 events per 100 patient years, respectively. The Kaplan-Meier survival curves showed that non-CW morphology was associated with embolic stroke $(p=0.021$, Figure 3A) and ischemic stroke ( $p$ $=0.040$, Figure 3B) recurrences in patients with ESUS and atrial cardiopathy by log-rank test, respectively. However, the differences in the other secondary outcomes during follow-up were not significant between the two groups (Figures 3C,D).

Non-CW LAA morphology was associated with embolic stroke recurrence in ESUS and atrial cardiopathy (HR, 3.17; 95\% CI, 1.13-8.91; $p=0.029$ ) in univariate Cox proportional hazards regression analysis. Other variables, including age and $\mathrm{CHA}_{2} \mathrm{DS}_{2}$-VASc score, were also associated with embolic stroke recurrence. Non-CW LAA morphology was independently associated with the recurrence of embolic stroke in multivariable Cox regression models 1-3 (Table 3 and Supplementary Tables 1-3). Age score in model 1 was also independently associated with embolic stroke recurrence. However, no significant independent associations of nonCW morphology exist with secondary outcomes (Table 3 and Supplementary Tables 1-3). TEE parameters were available in
TABLE 1 | Baseline demographics and clinical characteristics.

\begin{tabular}{|c|c|c|c|c|}
\hline & $\begin{array}{c}\text { All } \\
(n=157)\end{array}$ & $\begin{array}{l}\text { Chicken } \\
\text { wing } \\
(n=81)\end{array}$ & $\begin{array}{c}\text { Non-chicken } \\
\text { wing } \\
(n=76)\end{array}$ & $P$ \\
\hline Age & $69.5 \pm 11.6$ & $68.7 \pm 12.5$ & $70.3 \pm 10.6$ & 0.365 \\
\hline Men & $91(58.0)$ & $46(56.8)$ & $45(59.2)$ & 0.759 \\
\hline Baseline NIHSS & $3.0(1.0-7.0)$ & $2.0(1.0-6.5)$ & $3.5(1.0-7.8)$ & 0.434 \\
\hline $\begin{array}{l}\text { Pre-stroke mRS, 0-1 } \\
(\%)\end{array}$ & $136(86.6)$ & $72(88.9)$ & $64(84.2)$ & 0.389 \\
\hline Hypertension & $96(61.1)$ & $44(54.3)$ & $52(68.4)$ & 0.070 \\
\hline Diabetes mellitus & $34(21.7)$ & $13(16.0)$ & $21(27.6)$ & 0.078 \\
\hline Hyperlipidemia & $55(35.0)$ & $22(27.2)$ & $33(43.4)$ & 0.033 \\
\hline Coronary artery disease & $16(10.2)$ & $7(8.6)$ & $9(11.8)$ & 0.508 \\
\hline Congestive heart failure & $8(5.1)$ & $3(3.7)$ & $5(6.6)$ & 0.485 \\
\hline Prior stroke or TIA & $17(10.8)$ & $8(9.9)$ & $9(11.8)$ & 0.692 \\
\hline Smoking & $40(25.5)$ & 24 (29.6) & $16(21.1)$ & 0.218 \\
\hline \multicolumn{5}{|l|}{$\begin{array}{l}\text { Potential embolic } \\
\text { sources }\end{array}$} \\
\hline $\begin{array}{l}\text { Arterial atherosclerotic } \\
\text { disease }\end{array}$ & $103(65.6)$ & $50(61.7)$ & $53(69.7)$ & 0.291 \\
\hline $\begin{array}{l}\text { Left ventricular } \\
\text { dysfunction }\end{array}$ & $21(13.4)$ & $9(11.1)$ & $12(15.8)$ & 0.389 \\
\hline Cardiac valve disease & $7(4.5)$ & $4(4.9)$ & $3(3.9)$ & 1.000 \\
\hline Patent foramen ovale & $38(24.2)$ & $23(28.4)$ & $15(19.7)$ & 0.206 \\
\hline Cancer & $17(10.8)$ & $6(7.4)$ & $11(14.5)$ & 0.154 \\
\hline $\begin{array}{l}\text { Number of potential } \\
\text { embolic sources }\end{array}$ & & & & 0.864 \\
\hline 0 & $25(15.9)$ & $14(17.3)$ & $11(14.5)$ & \\
\hline 1 & $88(56.1)$ & $44(54.3)$ & $44(57.9)$ & \\
\hline$\geq 2$ & $44(28.0)$ & $23(28.4)$ & $21(27.6)$ & \\
\hline CHA2DS2-VASc score & $4.4 \pm 1.5$ & $4.3 \pm 1.5$ & $4.6 \pm 1.6$ & 0.172 \\
\hline Treatment on discharge & & & & 0.604 \\
\hline No antithrombotic & $3(1.9)$ & $1(1.2)$ & $2(2.6)$ & \\
\hline Antiplatelet (single) & $33(21.0)$ & $17(21.0)$ & $16(21.1)$ & \\
\hline DAPT & $109(69.4)$ & $56(69.1)$ & $53(69.7)$ & \\
\hline Oral anticoagulants & $9(5.7)$ & $4(4.9)$ & $5(6.6)$ & \\
\hline $\begin{array}{l}\text { Antiplatelet(s) and oral } \\
\text { anticoagulants }\end{array}$ & $3(1.9)$ & $3(3.7)$ & $0(0.0)$ & \\
\hline
\end{tabular}

Variables are presented as mean \pm standard deviation or absolute number (proportions). NIHSS, National Institute of Health Stroke Scale; mRS, modified Rankin Scale; TIA, transient ischemic attack; DAPT, dual antiplatelet therapy.

47 patients. The proportion of LAA flow velocity $<40 \mathrm{~cm} / \mathrm{s}$ was similar in patients with non-CW LAA morphology and those with CW morphology (11.8\% [2/17] vs. $10.0 \%$ [3/30]; $p=1.000)$. However, patients with non-CW LAA morphology showed the presence of SEC in LAA more frequently than those with CW morphology, albeit not statistically significant (52.9\% [9/17] vs. $43.3 \%[13 / 30] ; p=0.526)$.

\section{DISCUSSION}

The present study showed that the prevalence of individual LAA morphology types in ESUS and atrial cardiopathy were $51.6 \% \mathrm{CW}$ and $48.4 \%$ non-CW (21\% windsock, $15.9 \%$ cactus, 
TABLE 2 | Comparisons of cardiac markers between patients with and without chicken wing left atrial appendage morphology.

\begin{tabular}{|c|c|c|c|c|}
\hline & $\begin{array}{c}\text { All } \\
(n=157)\end{array}$ & $\begin{array}{l}\text { Chicken wing } \\
\quad(n=81)\end{array}$ & $\begin{array}{l}\text { Non-chicken wing } \\
\qquad(n=76)\end{array}$ & $P$ \\
\hline \multicolumn{5}{|l|}{ Echocardiographic findings } \\
\hline LA size (mm) & $40.7 \pm 5.8$ & $40.4 \pm 6.3$ & $41.0 \pm 5.2$ & 0.507 \\
\hline LV EF (\%) & $60.4 \pm 5.7$ & $60.3 \pm 5.9$ & $60.6 \pm 5.4$ & 0.773 \\
\hline LAA filling defect & $43(27.4)$ & $25(30.9)$ & $18(23.7)$ & 0.313 \\
\hline
\end{tabular}

Variables are presented as mean \pm standard deviation or absolute number (proportions).

$L A$, left atrium; EF, ejection fraction; LAA, left atrial appendage; AF, atrial fibrillation.

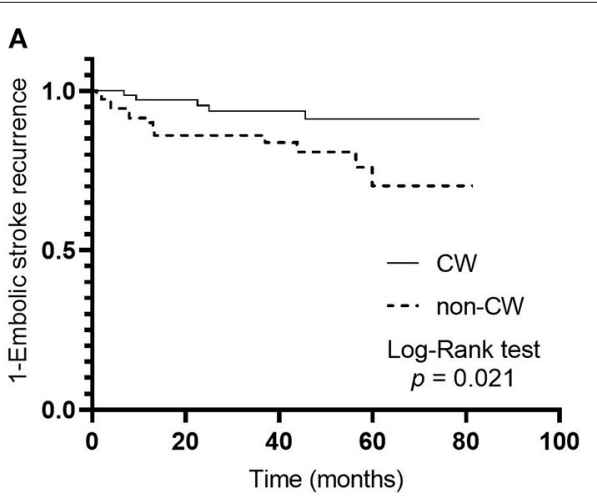

C

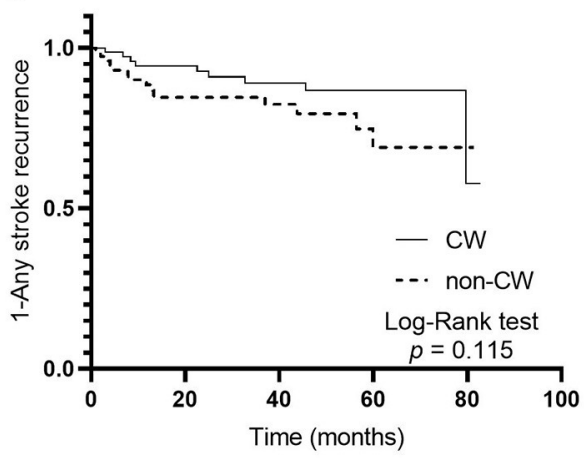

B

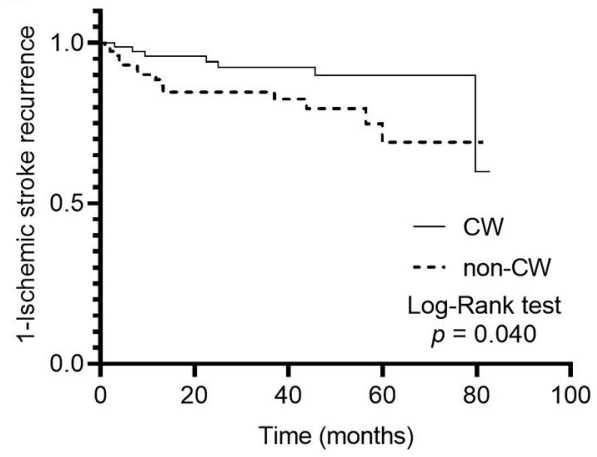

D

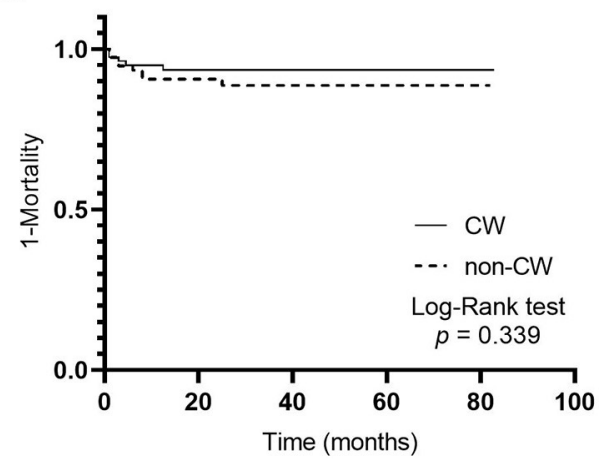

FIGURE 3 | Cumulative probability of primary and secondary outcomes in patients with embolic stroke of undetermined source and atrial cardiopathy according to left atrial appendage morphology; embolic stroke recurrence (A), ischemic stroke recurrence (B), any stroke recurrence (C), and mortality (D).

and $11.5 \%$ cauliflower). The event rates of embolic stroke recurrence, ischemic stroke recurrence, any stroke recurrence, and mortality in this population were $3.80,4.47,4.90$, and 2.55 per 100 patient years, respectively. Furthermore, the risk of embolic stroke recurrence in ischemic stroke patients with ESUS and atrial cardiopathy was independently associated with non-CW LAA morphology, though the associations of non-CW LAA morphology with the recurrence of any ischemic stroke, hemorrhagic stroke, or mortality were not statistically significant.

Several studies regarding LAA morphology and thromboembolism in AF have shown an association of
non-CW morphology with an embolic event $(14,16,18)$. This could possibly be explained by complex LAA morphology, characterized by extensive LAA trabeculations or increased number of lobes that are more suitable for non-CW than CW morphology, which was more likely to induce blood stasis leading to thrombus formation $(16,28)$. Furthermore, complex LAA morphology was related to significant LAA dysfunction, including a high degree of SEC (28) and low LAA emptying velocity (29). However, this association has seldom been investigated in the ESUS patient population with atrial cardiopathy. 
TABLE 3 | Univariate and multivariable Cox proportional hazards regression analyses investigating associations between non-chicken wing left atrial appendage morphology and outcomes.

\begin{tabular}{|c|c|c|c|c|}
\hline \multirow[t]{2}{*}{ Outcome $^{a}$} & $\begin{array}{c}\text { Crude HR } \\
(95 \% \mathrm{Cl})\end{array}$ & $\begin{array}{l}\text { Adjusted HR } \\
\qquad(95 \% \mathrm{Cl})\end{array}$ & $\begin{array}{l}\text { Adjusted HR } \\
\qquad(95 \% \mathrm{Cl})\end{array}$ & $\begin{array}{l}\text { Adjusted HR } \\
\text { (95\% Cl) }\end{array}$ \\
\hline & & Model $1^{b}$ & Model $2^{c}$ & Model $3^{d}$ \\
\hline Embolic stroke recurrence & $3.17(1.13-8.91)$ & $3.10(1.10-8.73)$ & $2.90(1.02-8.23)$ & $2.95(1.03-8.43)$ \\
\hline Ischemic stroke recurrence & $2.52(1.01-6.29)$ & $2.39(0.96-5.95)$ & $2.33(0.93-5.84)$ & $2.31(0.91-5.83)$ \\
\hline Any stroke recurrence & $1.95(0.84-4.52)$ & $1.85(0.80-4.29)$ & $1.60(0.70-3.64)$ & $1.58(0.69-3.63)$ \\
\hline Mortality & $1.71(0.56-5.22)$ & $1.56(0.50-4.91)$ & $1.66(0.54-5.11)$ & $1.44(0.44-4.71)$ \\
\hline
\end{tabular}

HR, hazard ratio; Cl, confidence interval.

a Outcomes are presented as the hazard ratio (95\% confidence interval) for values of non-chicken wing compared to chicken wing left atrial appendage morphology.

${ }^{b}$ Model 1 adjusted for age, sex, and baseline NIHSS score.

${ }^{c}$ Model 2 adjusted for $\mathrm{CH}_{2} D S_{2}$-VASc score and number of potential embolic sources.

${ }^{a}$ Model 3 adjusted for hypertension, diabetes mellitus, hyperlipidemia, and supraventricular tachycardia or subclinical atrial fibrillation.

The data in this study indicated that non-CW LAA morphology in patients with ESUS and atrial cardiopathy may also be associated with thrombus formation leading to the recurrence of embolic stroke. Non-CW LAA morphology tended to have more severe LAA dysfunction than CW morphology, although direct relationships could not be demonstrated due to a small number of patients whose TEE findings were available.

Identifying the factors associated with the high-risk of embolic stroke recurrence in patients with ESUS may aid secondary stroke prevention in clinical practice. Thus, patients with high-risk LAA morphology and ESUS with atrial cardiopathy may respond to anticoagulant rather than antiplatelet agents given the parallel results of the impact of non-CW LAA morphology on stroke events in ESUS with atrial cardiopathy and AF. Further studies are needed to prove the hypothesis.

This study has several limitations. Although several prespecified cox proportional hazards models were tested, the results may not be reliably examined due to the small sample size and limited number of outcome events. Selection bias and unregistered confounding factors cannot be ruled out due to its retrospective study design. Additionally, other potential biomarkers for atrial cardiopathy such as atrial fibrosis (6), P-wave terminal force in lead V1 on ECG (5), and N-terminal pro-brain natriuretic peptide (30) were not analyzed because of lacking data; therefore, interactions and degree of overlap among these biomarkers could not be evaluated. Moreover, the underlying pathophysiologic mechanisms of association between non-CW LAA morphology and the subsequent embolic event cannot be demonstrated because LAA dysfunctions including LAA flow velocity and SEC were evaluated in only a small subgroup of the study participants. Furthermore, the AF detection rate during the median follow-up period of 3.5 years was only $8.9 \%$ although all study participants underwent cardiac monitoring over $24 \mathrm{~h}$. The patients with paroxysmal AF or late-onset $\mathrm{AF}$ may be underestimated in this study considering the relatively low detection rate compared with other studies
$(31,32)$. Therefore, the association of high-risk LAA morphology with later detection of AF after an index stroke event could not be investigated.

In conclusion, the LAA morphology types may help identify the high risk of embolic stroke recurrence in ESUS with atrial cardiopathy. This biomarker in atrial cardiopathy may provide clues for selecting patients who may benefit from anticoagulants and developing therapies tailored to specific mechanisms.

\section{DATA AVAILABILITY STATEMENT}

The raw data supporting the conclusions of this article will be made available by the authors, without undue reservation.

\section{ETHICS STATEMENT}

The studies involving human participants were reviewed and approved by Kyungpook National University Hospital. Written informed consent for participation was not required for this study in accordance with the national legislation and the institutional requirements.

\section{AUTHOR CONTRIBUTIONS}

D-SG established the study protocol, analyzed and interpreted the data, and wrote the manuscript. WCC, Y-WK, and Y-SK analyzed the data. Y-HH established the study idea, interpreted the data, drafted the manuscript, and made critical revisions in the manuscript with intellectual input.

\section{SUPPLEMENTARY MATERIAL}

The Supplementary Material for this article can be found online at: https://www.frontiersin.org/articles/10.3389/fneur. 2021.679320/full\#supplementary-material 


\section{REFERENCES}

1. Hart RG, Diener HC, Coutts SB, Easton JD, Granger CB, O'donnell MJ, et al. Embolic strokes of undetermined source: the case for a new clinical construct. Lancet Neurol. (2014) 13:429-38. doi: 10.1016/S1474-4422(13)70310-7

2. Hart RG, Sharma M, Mundl H, Kasner SE, Bangdiwala SI, Berkowitz SD, et al. Rivaroxaban for stroke prevention after embolic stroke of undetermined source. N Engl J Med. (2018) 378:2191-201. doi: 10.1056/NEJMoa1802686

3. Diener HC, Sacco RL, Easton JD, Granger CB, Bernstein RA, Uchiyama S, et al. Dabigatran for prevention of stroke after embolic stroke of undetermined source. N Engl J Med. (2019) 380:1906-17. doi: 10.1056/NEJMoa1813959

4. Kamel H, Merkler AE, Iadecola C, Gupta A, Navi BB. Tailoring the approach to embolic stroke of undetermined source: a review. JAMA Neurol. (2019) 76:855-61. doi: 10.1001/jamaneurol.2019.0591

5. Kamel H, Soliman EZ, Heckbert SR, Kronmal RA, Longstreth WT Jr, Nazarian S, et al. P-wave morphology and the risk of incident ischemic stroke in the multi-ethnic study of atherosclerosis. Stroke. (2014) 45:27868. doi: 10.1161/STROKEAHA.114.006364

6. Goldberger JJ, Arora R, Green D, Greenland P, Lee DC, Lloyd-Jones DM, et al. Evaluating the atrial myopathy underlying atrial fibrillation: identifying the arrhythmogenic and thrombogenic substrate. Circulation. (2015) 132:27891. doi: 10.1161/CIRCULATIONAHA.115.016795

7. Marcus GM, Dewland TA.Premature atrial contractions: a wolf in sheep's clothing? J Am Coll Cardiol. (2015) 66:242-4. doi: 10.1016/j.jacc.2015.04.069

8. Hirsh BJ, Copeland-Halperin RS, Halperin JL. Fibrotic atrial cardiomyopathy, atrial fibrillation, and thromboembolism: mechanistic links and clinical inferences. J Am Coll Cardiol. (2015) 65:2239-51. doi: 10.1016/j.jacc.2015.03.557

9. Kamel H, Okin PM, Elkind MS, Iadecola C. Atrial fibrillation and mechanisms of stroke: time for a new model. Stroke. (2016) 47:895900. doi: 10.1161/STROKEAHA.115.012004

10. Kamel H, Bartz TM, Elkind MSV, Okin PM, Thacker EL, Patton KK, et al. Atrial cardiopathy and the risk of ischemic stroke in the CHS (Cardiovascular Health Study). Stroke. (2018) 49:980-6. doi: 10.1161/STROKEAHA.117.020059

11. Edwards JD, Healey JS, Fang J, Yip K, Gladstone DJ. Atrial cardiopathy in the absence of atrial fibrillation increases risk of ischemic stroke, incident atrial fibrillation, and mortality and improves stroke risk prediction. J Am Heart Assoc. (2020) 9:e013227. doi: 10.1161/JAHA.119.013227

12. Healey JS, Gladstone DJ, Swaminathan B, Eckstein J, Mundl H, Epstein $\mathrm{AE}$, et al. Recurrent stroke with rivaroxaban compared with aspirin according to predictors of atrial fibrillation: secondary analysis of the NAVIGATE ESUS randomized clinical trial. JAMA Neurol. (2019) 76:76473. doi: 10.1001/jamaneurol.2019.0617

13. Longstreth WT Jr, Kronmal RA, Thompson JL, Christenson RH, Levine SR, Gross R, et al. Amino terminal pro-B-type natriuretic peptide, secondary stroke prevention, and choice of antithrombotic therapy. Stroke. (2013) 44:714-9. doi: 10.1161/STROKEAHA.112.675942

14. Di Biase L, Santangeli P, Anselmino M, Mohanty P, Salvetti I, Gili S, et al. Does the left atrial appendage morphology correlate with the risk of stroke in patients with atrial fibrillation? results from a multicenter study. J Am Coll Cardiol. (2012) 60:531-8. doi: 10.1016/j.jacc.2012.04.032

15. Wang Y, Di Biase L, Horton RP, Nguyen T, Morhanty P, Natale A. Left atrial appendage studied by computed tomography to help planning for appendage closure device placement. J Cardiovasc Electrophysiol. (2010) 21:973-82. doi: 10.1111/j.1540-8167.2010.01814.x

16. Kimura T, Takatsuki S, Inagawa K, Katsumata Y, Nishiyama T, Nishiyama $\mathrm{N}$, et al. Anatomical characteristics of the left atrial appendage in cardiogenic stroke with low CHADS2 scores. Heart Rhythm. (2013) 10:9215. doi: 10.1016/j.hrthm.2013.01.036

17. Blackshear JL, Odell JA. Appendage obliteration to reduce stroke in cardiac surgical patients with atrial fibrillation. Ann Thorac Surg. (1996) 61:7559. doi: 10.1016/0003-4975(95)00887-X

18. Lee JM, Seo J, Uhm JS, Kim YJ, Lee HJ, Kim JY, et al. Why is left atrial appendage morphology related to strokes? an analysis of the flow velocity and orifice size of the left atrial appendage. J Cardiovasc Electrophysiol. (2015) 26:922-7. doi: 10.1111/jce.12710

19. Yaghi S, Kamel H, Elkind MSV. Atrial cardiopathy: a mechanism of cryptogenic stroke. Expert Rev Cardiovasc Ther. (2017) 15:5919. doi: 10.1080/14779072.2017.1355238

20. Ntaios G, Pearce LA, Veltkamp R, Sharma M, Kasner SE, Korompoki E, et al. Potential embolic sources and outcomes in embolic stroke of undetermined source in the NAVIGATE-ESUS Trial. Stroke. (2020) 51:1797804. doi: 10.1161/STROKEAHA.119.028669

21. Ntaios G, Perlepe K, Lambrou D, Sirimarco G, Strambo D, Eskandari A, et al. Prevalence and overlap of potential embolic sources in patients with embolic stroke of undetermined source. J Am Heart Assoc. (2019) 8:e012858. doi: 10.1161/JAHA.119.012858

22. Geisler T, Poli S, Meisner C, Schreieck J, Zuern CS, Nagele T, et al. Apixaban for treatment of embolic stroke of undetermined source (ATTICUS randomized trial): rationale and study design. Int J Stroke. (2017) 12:98590. doi: $10.1177 / 1747493016681019$

23. Teunissen C, Habets J, Velthuis BK, Cramer MJ, Loh P. Double-contrast, single-phase computed tomography angiography for ruling out left atrial appendage thrombus prior to atrial fibrillation ablation. Int $J$ Cardiovasc Imaging. (2017) 33:121-8. doi: 10.1007/s10554-016-0973-2

24. Ntaios G, Vemmos K, Lip GY, Koroboki E, Manios E, Vemmou A, et al. Risk stratification for recurrence and mortality in embolic stroke of undetermined source. Stroke. (2016) 47:2278-85. doi: 10.1161/STROKEAHA.116.013713

25. Lip GY, Nieuwlaat R, Pisters R, Lane DA, Crijns HJ. Refining clinical risk stratification for predicting stroke and thromboembolism in atrial fibrillation using a novel risk factor-based approach: the euro heart survey on atrial fibrillation. Chest. (2010) 137:263-72. doi: 10.1378/chest.09-1584

26. Delacretaz E. Clinical practice: supraventricular tachycardia. N Engl J Med. (2006) 354:1039-51. doi: 10.1056/NEJMcp051145

27. Beppu S, Nimura Y, Sakakibara H, Nagata S, Park YD, Izumi S. Smoke-like echo in the left atrial cavity in mitral valve disease: its features and significance. J Am Coll Cardiol. (1985) 6:744-9. doi: 10.1016/S0735-1097(85)80476-9

28. Yamamoto M, Seo Y, Kawamatsu N, Sato K, Sugano A, Machino-Ohtsuka T, et al. Complex left atrial appendage morphology and left atrial appendage thrombus formation in patients with atrial fibrillation. Circ Cardiovasc Imaging. (2014) 7:337-43. doi: 10.1161/CIRCIMAGING.113.001317

29. Fukushima K, Fukushima N, Kato K, Ejima K, Sato H, Fukushima K, et al. Correlation between left atrial appendage morphology and flow velocity in patients with paroxysmal atrial fibrillation. Eur Heart J Cardiovasc Imaging. (2016) 17:59-66. doi: 10.1093/ehjci/jev117

30. Cushman M, Judd SE, Howard VJ, Kissela B, Gutierrez OM, Jenny NS, et al. N-terminal pro-B-type natriuretic peptide and stroke risk: the reasons for geographic and racial differences in stroke cohort. Stroke. (2014) 45:164650. doi: 10.1161/STROKEAHA.114.004712

31. Gladstone DJ, Spring M, Dorian P, Panzov V, Thorpe KE, Hall $\mathrm{J}$, et al. Atrial fibrillation in patients with cryptogenic stroke. $N$ Engl J Med. (2014) 370:2467-77. doi: 10.1056/NEJMoa13 11376

32. Sanna T, Diener HC, Passman RS, Di Lazzaro V, Bernstein RA, Morillo CA, et al. Cryptogenic stroke and underlying atrial fibrillation. N Engl J Med. (2014) 370:2478-86. doi: 10.1056/NEJMoa13 13600

Conflict of Interest: The authors declare that the research was conducted in the absence of any commercial or financial relationships that could be construed as a potential conflict of interest.

Copyright $\odot 2021$ Gwak, Choi, Kim, Kim and Hwang. This is an open-access article distributed under the terms of the Creative Commons Attribution License (CC BY). The use, distribution or reproduction in other forums is permitted, provided the original author(s) and the copyright owner(s) are credited and that the original publication in this journal is cited, in accordance with accepted academic practice. No use, distribution or reproduction is permitted which does not comply with these terms. 